\title{
All-Optical 3R Regeneration Using Fiber Optical Parametric Oscillator and Amplifier
}

\author{
Xie Wang, Jianbing Xu, and Kenneth K. Y. Wong* \\ Photonic Systems Research Laboratory, Department of Electrical and Electronic Engineering, \\ The University of Hong Kong, Pokfulam Road, Hong Kong. \\ *E-mail:kywong@eee.hku.hk
}

\begin{abstract}
We demonstrate an all-optical wavelength-tunable 3R regenerator based on fiber optical parametric oscillator and amplifier. Regenerated signal with a power penalty of $0.1 \mathrm{~dB}$ versus back-to-back at $10^{-9}$ BER can be obtained.

(C)2012 Optical Society of America

OCIS codes: (070.4340) Nonlinear optical signal processing; (190.4970) Parametric oscillators and amplifiers.
\end{abstract}

\section{Introduction}

Optical signal propagation in high-speed optical communication networks usually suffers from much impairment caused by dispersion, nonlinearity and noise. All these effects limit the transmission distance and 3R (retiming, reshaping, and reamplification) regeneration is highly desirable to achieve long-distance transmission of high-speed signal. Different 3R regeneration schemes are demonstrated based on parametric amplification in fiber [1, 2], crossgain or cross-phase modulation in semiconductor optical amplifier (SOA) [3], cross-absorption modulation in electro-absorption modulator [4]. However, most of these schemes require optical-electronic-optical (OEO) module, which usually includes relatively expensive high-speed photodetector (PD), complicated electrical signal processing with limited electrical bandwidth and/or mode-locked laser diode. While in this paper, a cost-effective, all-fiber 3R regenerator based on fiber optical parametric oscillator (FOPO) and amplifier (FOPA) has been demonstrated. Due to the nature of FOPO and FOPA, our scheme has intrinsically ultra-fast response time and inherent parametric gain. Our scheme successfully achieves the improvement of timing jitter, signal-to-noise ratio (SNR) and extinction ratio over the tuning range of at least $8 \mathrm{~nm}$. We can obtain the regenerated signal with a power penalty of $0.1 \mathrm{~dB}$ compared to back-to-back signal and a receiver sensitivity improvement of $1.7 \mathrm{~dB}$ compared to degraded signal at $10^{-9}$ bit-error rate (BER) level. Furthermore, the chromatic dispersion tolerance of our scheme has been investigated.

\section{Experimental Setup}

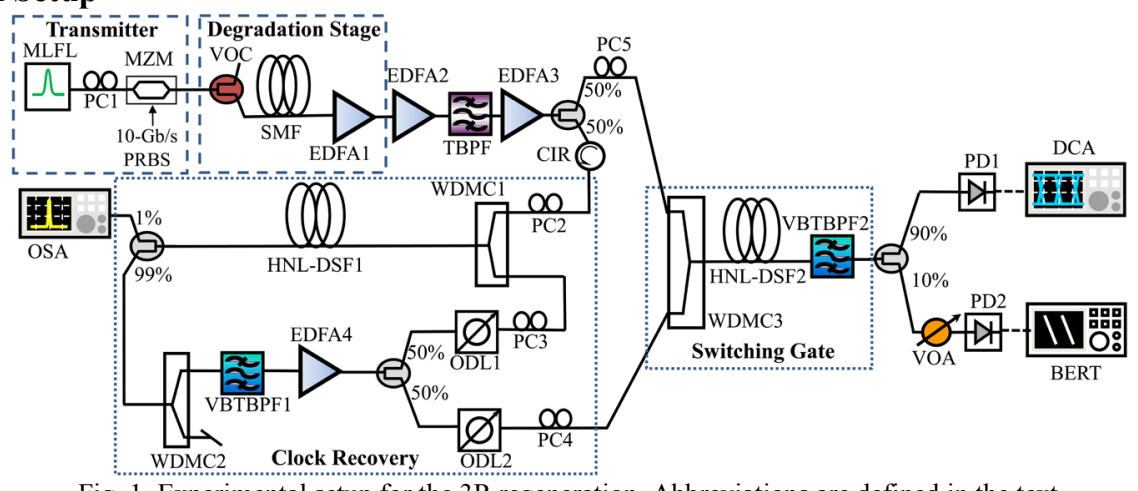

Fig. 1. Experimental setup for the 3R regeneration. Abbreviations are defined in the text.

Our 3R regenerator consists of a wavelength-tunable clock recovery and an optical switching gate. The clock recovery part which is based on FOPO can generate a low-noise, low timing jitter clock train [5]. By synchronizing this clock train with the degraded signal in a data-pumped FOPA which acts as an optical switching gate, the $3 \mathrm{R}$ regeneration can be obtained. Moreover, due to the quadratic relationship between the input signal power and the converted signal power in the data-pumped FOPA, the extinction ratio of the input signal is also improved.

The experimental setup is shown in Fig. 1. The 10-Gb/s return-to-zero (RZ) data signal with pulsewidth of 9.6 ps or $4.8 \mathrm{ps}$ at $1556.4 \mathrm{~nm}$ was generated by modulating the pulse train from a mode-locked fiber laser (MLFL) with a Mach-Zehnder modulator (MZM) which was driven by a $10-\mathrm{Gb} / \mathrm{s} 2^{31}-1$ pseudorandom binary sequence (PRBS). Then, the $10-\mathrm{Gb} / \mathrm{s}$ data signal passed the optical degradation stage which comprised a variable optical coupler (VOC) to control the input power, 3-km or 6-km single-mode fiber (SMF) which has a dispersion of $17 \mathrm{ps} / \mathrm{nm} / \mathrm{km}$ and a dispersion slope of $0.06 \mathrm{ps} / \mathrm{nm}^{2} / \mathrm{km}$ at $1550 \mathrm{~nm}$ to control the dispersive broadening, and an erbium-doped fiber 
amplifier (EDFA1) to add amplified spontaneous emission (ASE) noise. Afterwards, the degraded signal was launched into the 3R regeneration stage. The degraded signal was firstly amplified by EDFA2 and EDFA3, and filtered by a tunable bandpass filter (TBPF) to produce a high-power, low-noise pump for clock recovery and optical switching gate. In the clock recovery part, the amplified degraded signal then passed through a circulator, and was coupled into the cavity for parametric oscillation through the wavelength-division multiplexing coupler (WDMC1). Note that the cavity included an EDFA4 and 50-m highly-nonlinear dispersion-shifted fiber (HNL-DSF1) as the gain medium, the HNL-DSF1 has nonlinear coefficient of $14 \mathrm{~W}^{-1} \mathrm{~km}^{-1}$, zero-dispersion wavelength (ZDW) of $1554.7 \mathrm{~nm}$, and dispersion slope of $0.035 \mathrm{ps} / \mathrm{nm}^{2} / \mathrm{km}$. The FOPO output spectrum was monitored by an optical spectrum analyzer (OSA) through a 99/1 coupler, while the WDMC2 was used to block the pump. The recovered clock was filtered out by the variable bandwidth tunable bandpass filter (VBTBPF1). Its wavelength can be tuned by adjusting the center wavelength of VBTBPF1. The optical delay line (ODL1) in the cavity was used to synchronize the clock with the pump. The polarization controller PC2 and PC3 were used to align the state-of-polarization (SOP) of the clock with that of the pump. A 50/50 coupler in the cavity provided $50 \%$ feedback and $50 \%$ output. In the switching gate part, the ODL2 in the clock branch was used to align the clock pulse train with the degraded 10-Gb/s signal. PC4 and PC5 were used to align the SOP of the clock with that of the degraded signal so as to maximize the parametric gain. The clock and the degraded signal were combined by WDMC3 and launched into a 400-m HNLDSF2 for parametric amplification, which has the same nonlinear coefficient, ZDW and dispersion slope as the HNL-DSF1. After the parametric amplification in the HNL-DSF2, VBTBPF2 with a bandwidth of $5 \mathrm{~nm}$ was used to filter out the regenerated signal in the L-band. The waveform of the regenerated signal was recorded using a digital communication analyzer (DCA) and the performance was quantified by a bit-error rate tester (BERT).

\section{Results and Discussion}

Firstly, we used 9.6-ps pulsewidth 10-Gb/s RZ data signal at $1556.4 \mathrm{~nm}, 3-\mathrm{km}$ SMF, and introduced an offset bias on one arm of the MZM to control the signal extinction ratio. After passing through 3-km SMF, data signal with a 9.6-ps width was broadened to $23 \mathrm{ps}$. The extinction ratio and SNR of the degraded signal were measured to be 8.33 $\mathrm{dB}$ and $8.05 \mathrm{~dB}$, respectively. The average power of the pump for clock recovery and switching gate were both set to be $18 \mathrm{dBm}$. The clock we chose in this case had a center wavelength of $1544.5 \mathrm{~nm}$ with 6-ps pulsewidth and 10.7$\mathrm{dBm}$ average power measured after the 50/50 coupler inside the FOPO cavity. Then after passing through the optical switching gate, we can obtain the regenerated signal whose pulsewidth was narrower than the degraded signal at the wavelength of $1568.5 \mathrm{~nm}$. Its output power was only $4.6 \mathrm{dBm}$ (versus 9.1-dBm clock input power before HNLDSF2) due to the insertion loss of the VBTBPF2 $(\sim 5 \mathrm{~dB})$. Fig. 2(a) shows the optical spectra measured after the data-pumped FOPA when the degraded signal (pump) was switched off and on. The extinction ratio and SNR of the regenerated signal were improved to $15.21 \mathrm{~dB}$ and $13.5 \mathrm{~dB}$, respectively. Fig. 2(b) shows the measured eye diagram of the degraded signal and the regenerated signal. The performance of the $3 \mathrm{R}$ regenerator was further quantified by measuring the corresponding BER as shown in Fig. 2(b). A receiver sensitivity improvement of $1.7 \mathrm{~dB}$ was observed at $10^{-9}$ BER level.
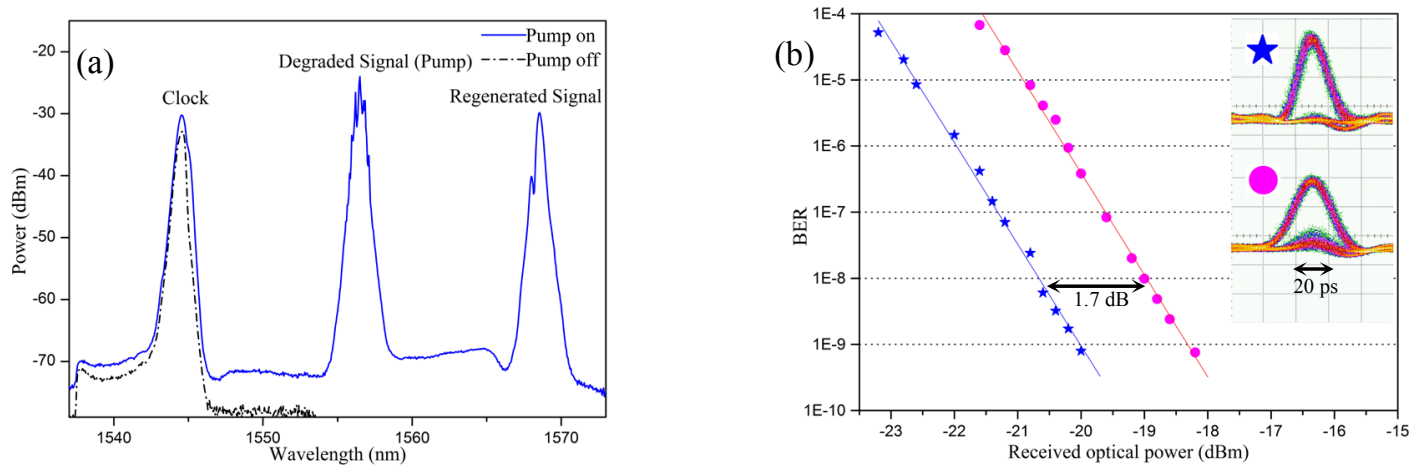

Fig. 2. (a) Optical spectra measured after the data-pumped FOPA when the pump was switched off (black dashed line) and on (blue solid line); (b) Measured eye diagram and BER of degraded signal (magenta circle) at $1556.4 \mathrm{~nm}$ and regenerated signal (blue star) at $1568.5 \mathrm{~nm}$.

Secondly, we changed the pulsewidth of $9.6 \mathrm{ps}$ to $4.8 \mathrm{ps}$ and replaced the 3-km SMF with 6-km SMF to further investigate the dispersion tolerance and wavelength tunability of our scheme. We still intentionally introduced an offset bias on the MZM. After the degradation stage, the $10-\mathrm{Gb} / \mathrm{s}$ RZ data signal was estimated to be broadened to $38 \mathrm{ps}$ and the RMS timing jitter, SNR and extinction ratio of the degraded signal were measured to be $2.78 \mathrm{ps}, 10.71$ $\mathrm{dB}$ and $9.63 \mathrm{~dB}$, respectively. The average power of the pump for clock recovery and switching gate was $20 \mathrm{dBm}$. 
The achieved wavelength tuning range of the clock was from $1541.3 \mathrm{~nm}$ to $1549.6 \mathrm{~nm}$ by simply adjusting the center wavelength of VBTBPF1, ODL1 and PC3 in the FOPO cavity. Fig. 3(a) shows the optical spectra measured after the 99/1 coupler inside the cavity. The wavelength of the regenerated signal can be tuned from $1563.4 \mathrm{~nm}$ to $1572 \mathrm{~nm}$, correspondingly. The improvement of timing jitter, SNR and extinction ratio achieved in this tuning range is shown in Table 1. Fig. 3(b) shows the BER measurement and the eye diagrams when the regenerated signal was at $1563.4 \mathrm{~nm}$. The back-to-back BER and eye diagram were measured right after the MZM when we did not introduce an offset bias. The power penalty of the regenerated signal compared to the back-to-back was $0.1 \mathrm{~dB}$ and the receiver sensitivity improvement compared to the degraded signal was $1.7 \mathrm{~dB}$ at $10^{-9} \mathrm{BER}$. When we measured the eye diagram of the signal whose pulsewidth was narrower than $10 \mathrm{ps}$, the pulsewidth observed in DCA was broadened due to the limited bandwidth $(30 \mathrm{GHz})$ of the PD1 before DCA.

Table 1. Measured RMS timing jitter, SNR and extinction ratio of the regenerated signal in the tuning range from

\begin{tabular}{c|l|l|l|l|l|l|l|l}
\hline \multicolumn{2}{c}{$1563.4 \mathrm{~nm}$ to $1572 \mathrm{~nm}}$. \\
\hline $\begin{array}{c}\text { Regenerated signal } \\
\text { wavelength (nm) }\end{array}$ & 1572 & 1571 & 1569.7 & 1568.5 & 1567 & 1565.5 & 1564.4 & 1563.4 \\
\hline RMS timing jitter (ps) & 2.09 & 2.05 & 2.11 & 1.995 & 2.10 & 2.02 & 1.846 & 2.04 \\
\hline Eye SNR (dB) & 12.15 & 14.01 & 13.89 & 14.42 & 13.95 & 11.72 & 13.09 & 14.63 \\
\hline Extinction Ratio (dB) & 17.91 & 17.66 & 17.45 & 16.01 & 16.13 & 16.46 & 16.78 & 15.86 \\
\hline
\end{tabular}

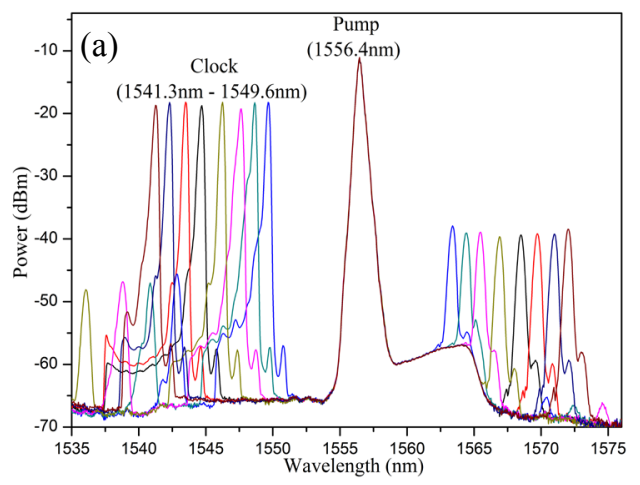

(b)

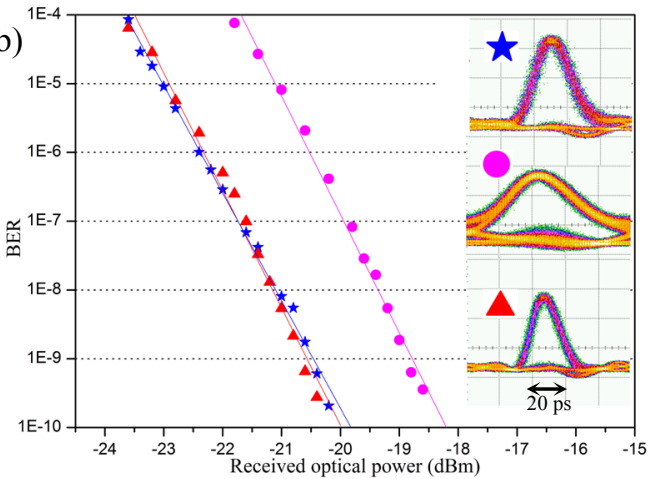

Fig. 3. (a) Optical spectra measured after the 99/1 coupler inside the FOPO cavity when tuning the clock output wavelength; (b) Measured eye diagram and BER of degraded signal (magenta circle) at $1556.4 \mathrm{~nm}$, regenerated signal (blue star) at $1563.4 \mathrm{~nm}$ and back-to-back signal (red triangle) at $1556.4 \mathrm{~nm}$.

\section{Conclusion}

In conclusion, we demonstrated a cost-effective, all-fiber 3R regenerator based on FOPO and FOPA. The tuning range of the regenerated signal was from $1563.4 \mathrm{~nm}$ to $1572 \mathrm{~nm}$ within which the enhancement of timing jitter, SNR and extinction ratio was achieved. Signal with $0.1-\mathrm{dB}$ power penalty at $10^{-9}$ BER can be regenerated at $1563.4 \mathrm{~nm}$. The chromatic dispersion broadening of the pulse can also be compensated partially. Our scheme has the potential to be extended to $40-\mathrm{Gb} / \mathrm{s}$ or higher speed due to the ultra-fast nature of the parametric process.

\section{Acknowledgement}

The work described in this paper was partially supported by grants from the Research Grants Council of the Hong Kong Special Administrative Region, China (Project No. HKU 7179/08E and 7183/09E). The authors would like to acknowledge Sumitomo Electric Industries for providing the HNL-DSF and the Alnair Labs Corporation for providing the VBTBPF.

\section{References}

[1] Y. Su, L. Wang, A. Agarwal, and P. Kumar, "Simultaneous 3R regeneration and wavelength conversion using a fiber-parametric limiting amplifier," in Proc. Optical Communication Conf., pp. MG4-1-MG4-3 (2001).

[2] C. Yu, T. Luo, B. Zhang, Z. Pan, M. Adler, Y. Wang, J. E. McGeehan, and A.E. Willner, "Wavelength-Shift-Free 3R Regenerator for 40-Gb/s RZ System by Optical Parametric Amplification in Fiber,” IEEE Photonics Technol. Lett., 18, 2569-2571 (2006).

[3] G. Gavioli, B. C. Thomsen, V. Mikhailov, and P. Bayvel, "Cascadability Properties of Optical 3R Regenerators Based on SOAs," J. Lightwave Technol., vol. 25, pp. 2766-2775 (2007).

[4] T. Otani, T. Miyazaki, and S. Yamamoto, "40-Gb/s Optical 3R Regenerator Using Electroabsorption Modulator for Optical Networks," J. Lightwave Technol., vol. 20, pp. 195-200 (2002).

[5] Y. Su, L. Wang, A. Agarwal, and P. Kumar, "Wavelength-tunable all-optical clock recovery using a fiber-optic parametric oscillator," Opt. Communications, 184, 151-156 (2000). 